\title{
Wearable Arm-Band Mobile ECG Monitoring System
}

\author{
Anshiq Nazar ${ }^{1}$, Abhijith PM$^{2}$, Mohammed Dhanish $\mathrm{KS}^{3}$, Junain Sidique ${ }^{4}$, Nuafil Ibrahim ${ }^{5}$, \\ Ms. Shahaziya Parveez \\ UG Scholar, Electronics and Communication, IES College of Engineering, Thrissur, India ${ }^{1,2,3,4,5}$ \\ Asst. Professor, Electronics and Communication, IES College of Engineering, Thrissur, India ${ }^{6}$
}

\begin{abstract}
One of the best ways to obtain health information is from an electrocardiogram (ECG). Through an ECG, characteristics such as patients' heartbeats, heart conditions, and heart disease can be analyzed. Unfortunately, most available healthcare devices do not provide clinical data such as information regarding patients' heart activities. Many researchers have tried to solve this problem by inventing wearable heart monitoring systems with a chest strap or wrist band, but their performances were not feasible for practical applications. Thus, the aim of this study is to build a new system to monitor heart activity through ECG signals. The proposed system consists of an embedded hardware in an armband. It is considered to be a reliable, robust, and low-power-transmission ECG monitoring system. The reliability of this system was achieved by the careful placement of sensors in the arm band. IoT is used as the protocol for data transmission.
\end{abstract}

Keywords: electrocardiogram, heart rate, blood pressure, PPG.

\section{INTRODUCTION}

Mobile health is a term that is used to describe healthcare services with the assistance of mobile devices. The M-health monitoring and diagnostic medical devices market is currently in its infancy but is expected to grow rapidly due to market demand for remote monitoring systems. According to a new report from Transparency Market Research the market for mobile health monitoring and diagnostics will grow at compound annual growth rate of 43.3 percent from 2013 to 2019. This will put the market at $\$ 8$ billion in 2019. Increased sports activities and awareness about health and fitness are some of the factors that are anticipated to fuel the growth of this market. Unfortunately, high demand does not guarantee that the devices available in the market are reliable and trustworthy.

Various studies have been conducted related to the development of remote healthcare systems, especially heart rate monitoring systems. Most of these studies have focused on four main topics: sensor technology, wearable systems, signal processing, and mobile monitoring systems. Researchers have tried to develop sensors that are able to sense biosignals with- out generating side effects or distracting users, but these were still unable to provide a high level of accuracy. The standard procedure in a hospital to measure an electrocardiogram (ECG) is to use a 12-lead ECG. In a conventional 12-lead clinical ECG system, electrodes are affixed to specific parts of the chest, arms, or hands and legs. Even though this promises highly accurate results, it often requires a great deal of preparation and an expert to attach the electrodes to the patient's body. These electrodes also require skin preparation and conduction gel to reduce contact impedance.. The main problem with this method is that it cannot be used for long-term measurement because of the limitation of the gel and complicated wiring.

Because of this, other techniques have been provided to over-come these weaknesses, such as using dry electrodes or noncontact electrodes. Dry electrodes consist of a metal with no electrolyte or conductive gel between the electrode and the skin. Instead, sweat or moisture on the skin will reduce the impedance between the skin and the electrodes. Various applications have been proposed using the dry-electrode technique, but these required direct contact with the skin. Thus, researchers have invented a noncontact or capacitive-coupled ECG. The capacitive-coupled ECG was first introduced by Lopez and Richardson.

Then, researchers expanded its use in various environments. Lim et al. and Yama et al. developed a heart monitoring system in office chairs and mattresses using flexible fabric electrodes. Leonhardt et al. introduced the idea of an insulated electrode implemented in a car system. Lee et al. proposed the use of thin and flexible electrodes for a wearable ECG system to build a system that can measure an ECG in remote areas. Oehler et al. proposed an integrated capacitive-electrode array and combined this array with a personal computer tablet. Different placements of sensors have been proposed by various researchers. Some have suggested using belt-type ECG monitoring systems with the 
implementation of conductive fabric and active electrodes. Others have suggested using bio-clothes for vital sign monitoring.

Different placements would provide different shapes of the ECG signal; thus, they tried to find the best place from which to measure an ECG based on demand. Nevertheless, researchers also focused on developing a mobile monitoring system. This system uses bio-signals to analyse health conditions with a mobile device as an interface and an analysis device. Several applications have been researched and proposed by other researchers in for example, to monitor HRV for vehicle drivers, detect arrhythmia, observe sleep disorders, and monitor driver fatigue.

Therefore, capacitive-coupled technology is used in the proposed system to overcome these problems. It is a promising method to measure an ECG in remote areas without inconveniencing or disturbing the user. The advantages of this technique are that it can measure an ECG without direct contact and provides better accuracy than other methods, especially for measuring stress. The proposed monitoring system is important for monitoring exercise intensity, estimation of maximal oxygen uptake and energy expenditure and early detection and in helping keep persons healthy by being able to track their heart activities at any time. Currently, most systems that are available in the market use a chest strap or a wristband, but this system is uncomfortable and requires direct contact with the skin. Consequently, an armband was chosen as an alternative to this inconvenient system. The proposed armband ECG is equipped with a variety of features. Considering mobile monitoring, it is equipped with an Android mobile application and Bluetooth low energy (BLE) data transmission. Real-time heart rate detection was added to the Android application, and this was tested in various scenarios, from sitting in a chair, standing, and walking to jogging or running in place.

\section{EXISTING SYSTEM}

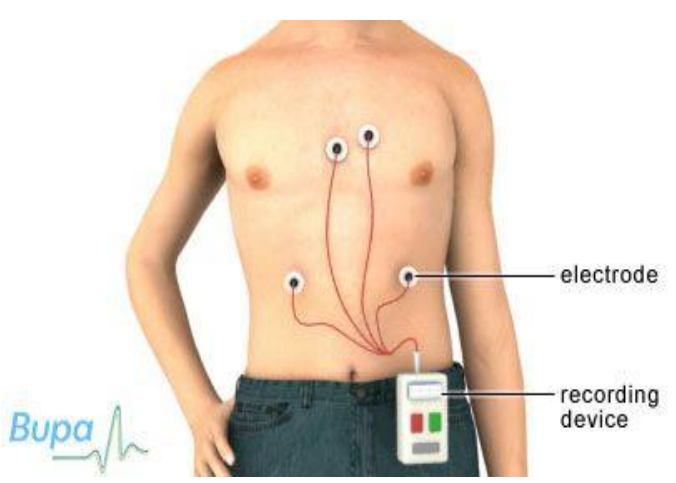

Fig.3.1 Electrodes with recording device

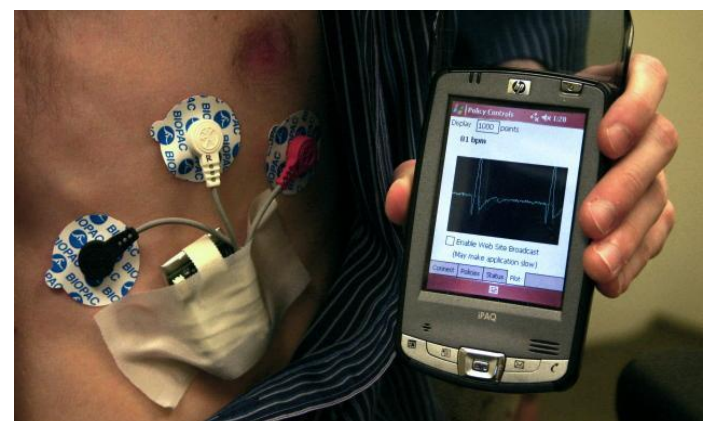

Fig.3.2 Electrodes connected to a smart phone

ECG (electrocardiogram) is currently the most effective way to detect the heart condition. Previously people have to go to special organization, e.g. hospital, to do the ECG recording. But sometimes when the patient arrives at the hospital, the symptom has disappeared. This may loss the early treatment opportunity. In this project, we use the Android smartphone and a Bluetooth enabled device to acquire the ECG signal. When the user feels something wrong with the heart, he/she can do the ECG recording immediately. The device uses a conductive belt to measure the ECG signal. The measured signal is sent to the smartphone via Bluetooth. Normally the ECG waveform is displayed on the screen of the smartphone to monitor the heart condition. The user can store the ECG waveform for further investigation. The waveform can be stored on the SD card of the smartphone for short term storage. It can also be sent to the cloud computer for long term storage and be accessed globally via the Internet. For the heart disease patients, they can use 


\section{ISO 3297:2007 Certified}

Vol. 5, Issue 4, April 2017

this device to track the effect of the medicine by storing the ECG before and after taking the medicine. This can help the doctor to decide the effectiveness of the medicine as early as possible, and avoid the waste of medical resources. This device can also be used in real-time which is useful for remote medical care.

To evaluate the performance of the device, we use it to measure ECG and apply conductive textile as our ECG electrode. Then, we evaluate ECG signals captured by different electrodes, specifically traditional gel $\mathrm{Ag} / \mathrm{AgCl}$ and dry golden plate electrodes, and compare the results, allowing us to investigate if ECG measured with the device is proper for applications where no skin preparation is allowed, such as ECG-assisted blood pressure monitoring devices. Next, we propose a method to reconstruct the ECG signal from the signal acquired by our device, with respect to the interface characteristics and their relation to the ECG. The interface in this paper is skin-electrode interface for conductive textiles. In the last stage of this paper, we explore the effects of pressure on skin-electrode interface impedance and its parametrical variation.

\section{PROPOSED SYSTEM}

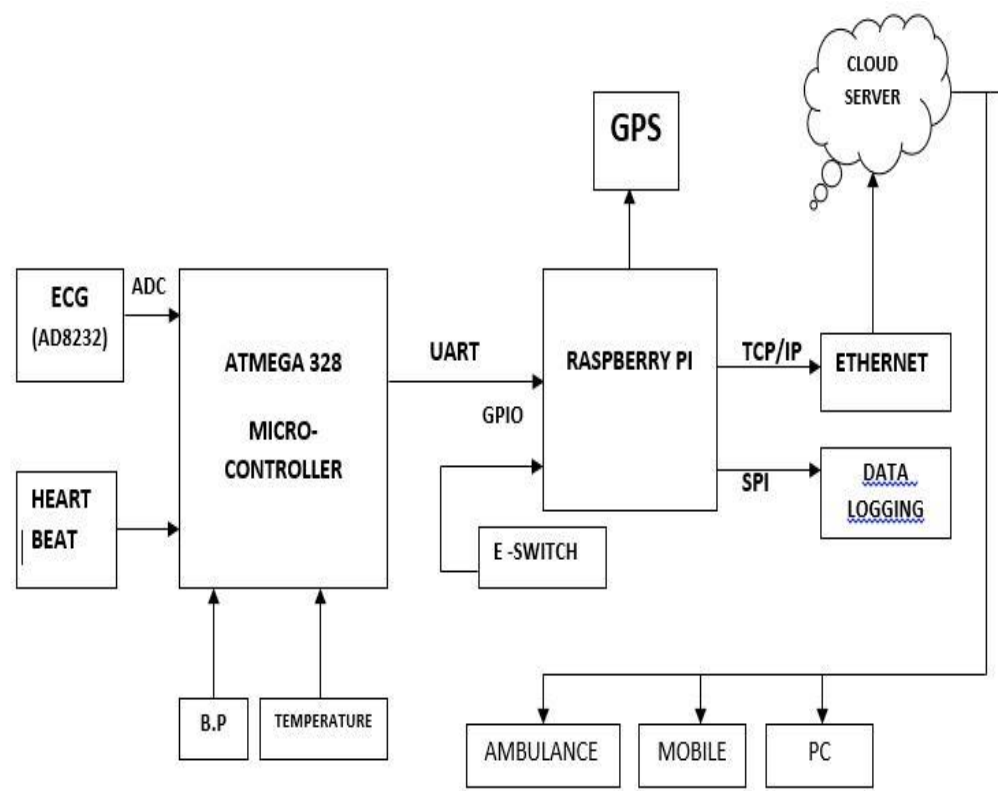

Fig 4.1 Block Diagram of the proposed system.

In this system we measure ECG using ECG sensor AD8232 that produces ADC output. Atmega328 is used as the controller. It processes the data from the sensor. Processed ECG raw value is send by using serial communication (UART) to the raspberry pi. Photo plethysmography (PPG) sensor is used as our heat beat sensor. When the detector is working, the beat LED flashes in unison with each heartbeat. The output is connected to the Atmega328 directly to measure the Beats per minute (BPM) rate. The systolic and diastolic pressure from the BP sensor, temperature measurement through LM35 sensor and heart beat are measured and processed by the controller and given to the raspberry pi. The raspberry pi processes these data where uploaded to the cloud server so a server named plotly is used to plot these data. Thus the measured data such as ECG, Temperature and heart beat rate can be monitored through the smart phones or PC with the help of Plotly. If any variations in the human reading it will alert authorities. The Eswitch can be used in case of any emergency.

\section{SOFTWARE DESCRIPTION}

The programming language used in this system is Python. Python is a widely used high-level, general purpose, interpreted, dynamic programming language. Its syntax allows programmers to express concepts in fewer lines of codes than possible in languages such as $\mathrm{C}++$ or Java. The language provide s constructs intended to enable writing clear programs on both a small and large scale. Python features a dynamic type system and automatic memory management and supports multiple management and supports multiple programming paradigms, including object-oriented, imperative, functional programming, and procedural styles. It has a large and comprehensive standard library. Python interpreters are available for many operating systems, allowing python code to run on a wide variety of systems. 


\section{ISO 3297:2007 Certified}

Vol. 5, Issue 4, April 2017

\section{COMPONENT DESCRIPTION}

\section{AD 8232 - ECG Sensor}

The AD8232 is a neat little chip used to measure the electrical activity of the heart. This electrical activity can be charted as an Electrocardiogram. Electrocardiography is used to help diagnose various heart conditions. The AD8232 Heart Rate Monitor breaks out nine connections from the IC. We traditionally call these connections "pins" because they come from the pins on the IC, but they are actually holes that you can solder wires or header pins to.We'll connect five of the nine pins on the board to your Arduino. The five pins you need are labeled GND, 3.3v, OUTPUT, $\mathrm{LO}-$, and LO+.

\section{LED Pulse Sensor (PPG) for Arduino}

Within this Instructable it is shown, how to implementsimplePPG(photoplethysmogram) sensor for Arduino. This device uses infrared light to measure you pulse. You may know this technique from the fingertip pulse sensors in hospitals. This techniques gets more and more important again in wearables like the apple watch theses days. The board i developed uses one wavelenghts to measure the pulse and within this, the measurement is used to control the LED brightness (which is important vor varying skin colors). The board can be powered by arduino or by its own voltage source. Three LEDs are controllable by the Arduinos digital outputs, for instance to show the pulse waves signal amplitude by software.

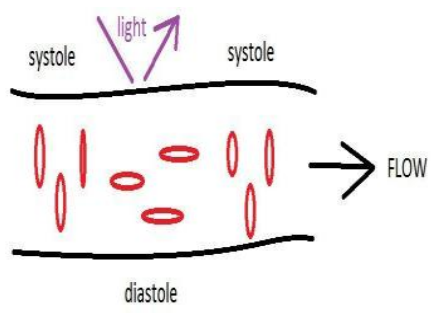

Fig 4.2 Blood flow

When blood is pumped through the body the pressure and consequentially the diameters of the vessels change. If the emitter and detector, both working by wavelengths which can pass the skin, are placed at a pulsating vessel, the path length of the light will periodically increase and decrease. This has the effect that also the absorption alternates. Due to simplification the emitter and detector are placed at the same side. Therefore, this method is based on reflection of the emitted light.

Next to the absorption of the blood cells, their flow behavior has an important role. The shown image indicates, that the blood cells move in a direction which are aligned orthogonal to the flow direction, when high flows are appearing. This is the case for the systolic phase. With this behavior, the reflective area of the blood cells de- and increase during the systolic and diastolic phase, which leads to an changing intensity of the reflected light.

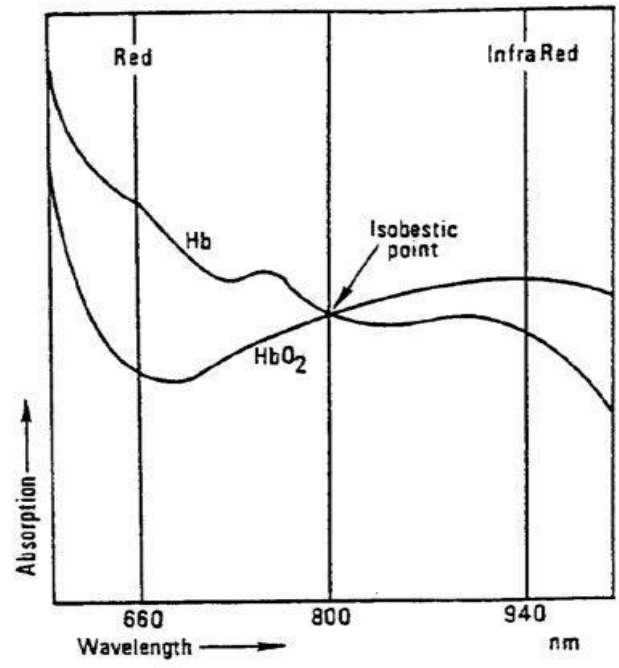

Fig 4.3 Absorptions for infrared light 
Fig 3.3 indicates that hemoglobin has different absorptions for infrared light, dependent on its oxygen load. This effects summed up are used to get a different signal amplitude of the reflected light if a pulse is measured. Welcome to the world of photoplethysmograms.

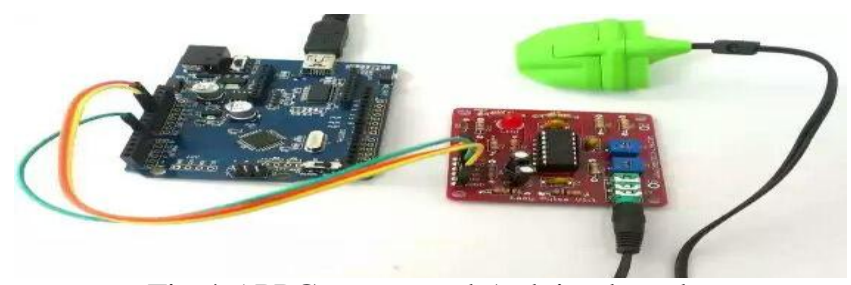

Fig 4.5 PPG sensor and Arduino board

The Signal one can get from such a device consists out of a DC part (skin/bone reflection, venous blood absorption, constant arterial blood absorption) and the wanted AC part (variable arterial blood absorption).

\section{Heart rate sensor AD8232 Interface Arduino}

The AD8232 from Analog Devices is a dedicated single lead heart rate monitor front end integrated circuit. The AD8232 is an integrated signal conditioning block for ECG and other bio potential measurement applications. It is designed to extract, amplify, and filter small bio potential signals in the presence of noisy conditions, such as those created by motion or remote electrode placement. This design allows for an ultralow power analog-to-digital converter (ADC) or an embedded microcontroller to acquire the output signal easily.

The simple and easy to use breakout board for heart rate monitoring from sparkfun. This board measures electrical activity of heart through the electrodes placed on the skin. By Interfacing this board with Arduino we can get ECG graph through Processing IDE window.

\section{Heart Monitor AD8232 Board}

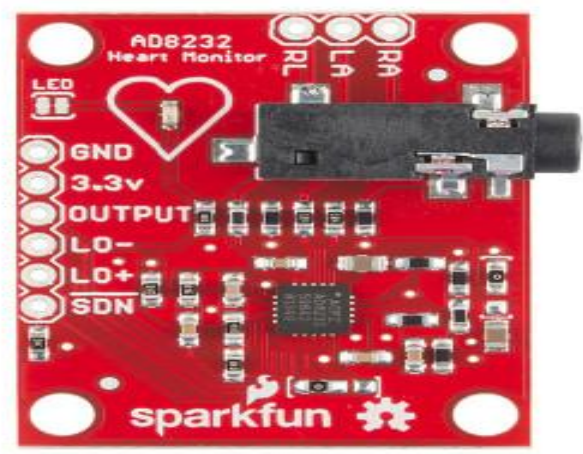

Fig 4.6 AD8232 Board

We can use the electrode jack or else pin holes for electrodes. Connect corresponding electrode pads in skin and then provide 3.3V and GND power supply from the Arduino board, the SDN (shutdown) pin is not connected to any part. Output from the breakout board is taken to Arduino's A0 (Analog input 0) pin. To detect the Leads off situation LO -, $\mathrm{LO}+$ are connected to Arduino digital pin D11 and D10 respectively.

\section{Blood Pressure Sensor}

Blood Pressure \& Pulse reading are shown on display with serial out for external projects of embedded circuit processing and display. Shows Systolic, Diastolic and Pulse Readings. Compact design fits over your wrist like a watch. Easy to use wrist style eliminates pumping.

\section{Temperature Sensor - LM35}

The LM35 series are precision integrated-circuit temperature sensors, whose output voltage is linearly proportional to the Celsius (Centigrade) temperature. The LM35 thus has an advantage over linear temperature sensors calibrated in $\S$ Kelvin, as the user is not required to subtract a large constant voltage from its output to obtain convenient Centigrade scaling. The LM35 does not require any external calibration or trimming to provide typical accuracies of $\mathrm{g}(/ 4 \S \mathrm{C}$ at 


\section{International Journal of Innovative Research in Electrical, Electronics, Instrumentation and Control Engineering}

\section{ISO 3297:2007 Certified}

Vol. 5, Issue 4, April 2017

room temperature and $\mathrm{g} * / 4 \S$ Cover a full b55 to a150§C temperature range. Low cost is assured by trimming and calibration at the wafer level. The LM35's low output impedance, linear output, and precise inherent calibration make interfacing to readout or control circuitry especially easy. It can be used with single power supplies, or with plus and minus supplies.

\section{Heart Beat Sensor}

Heart beat sensor is designed to give digital output of heat beat when a finger is placed on it. When the heart beat detector is working, the beat LED flashes in unison with each heart beat. This digital output can be connected to microcontroller directly to measure the Beats per Minute (BPM) rate. It works on the principle of light modulation by blood flow through finger at each pulse.

\section{Internet of Things}

The Internet of Things (IoTs) can be described as connecting everyday objects like smart-phones, Internet TVs, sensors and actuators to the World Wide Web where the devices are intelligently linked together enabling new forms of communication between things and people, and between things themselves. Building IoTs has advanced significantly in the last couple of years since it has added a new dimension to the world of information and communication technologies. In 2008, the number of connected devices surpassed connected people and it has been estimated by Cisco that by 2020 there will be 50 billion connected devices which is seven times the world population. Now anyone, from anytime and anywhere can have connectivity for anything and it is expected thatthese connections will extend and create an entirely advanced dynamic network of IoTs. The development of the Internet of Things will revolutionize a number of sectors, from wireless sensors to nanotechnology.

\section{WORKING}

In this system we measure ECG using ECG sensor AD8232 that produces ADC output. Atmega328 is used as the controller. It processes the data from the sensor. Processed ECG raw value is send by using serial communication (uart) to the raspberry pi. Photoplethysmography (PPG) sensor is used as our heat beat sensor. When the detector is working, the beat LED flashes in unison with each heartbeat. The output is connected to the Atmega328 directly to measure the Beats per minute (BPM) rate. The systolic and diastolic pressure from the BP sensor, temperature measurement through LM35 sensor and heart beat are measured and processed by the controller and given to the raspberry pi. The raspberry pi processes these data where uploaded to the cloud server so a server named plotly is used to plot these data. Thus the measured data such as ECG, Temperature and heart beat rate can be monitored through the smart phones or PC with the help of Plotly. If any variations in the human reading it will alert authorities. The E-switch can be used in case of any emergency.

\section{IX.RESULTS}

Thus we obtained the result of our project Wearable arm-band ECG mobile monitoring system.

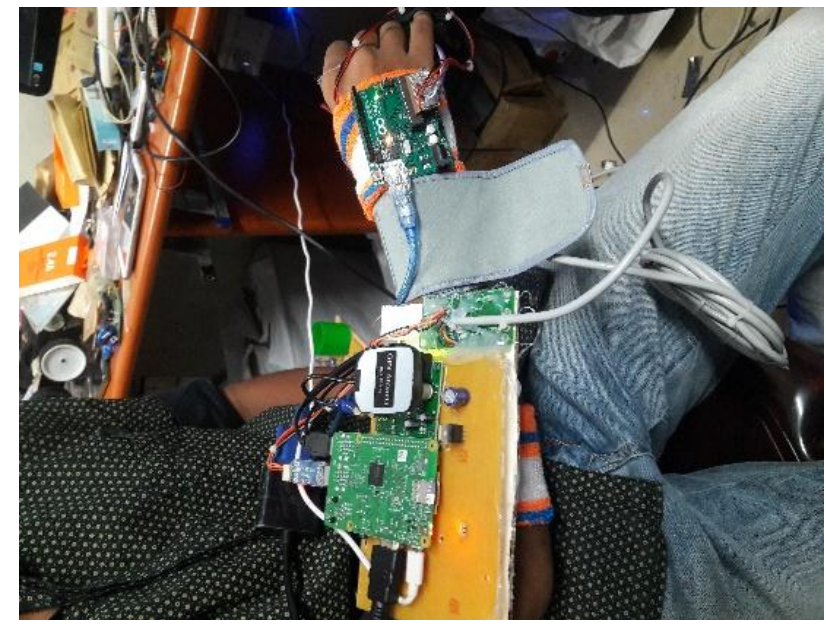

Fig 6.1 System prototype

The above shown is the prototype of our project Wearable arm-band ECG mobile monitoring system. 


\section{IJIREEICE
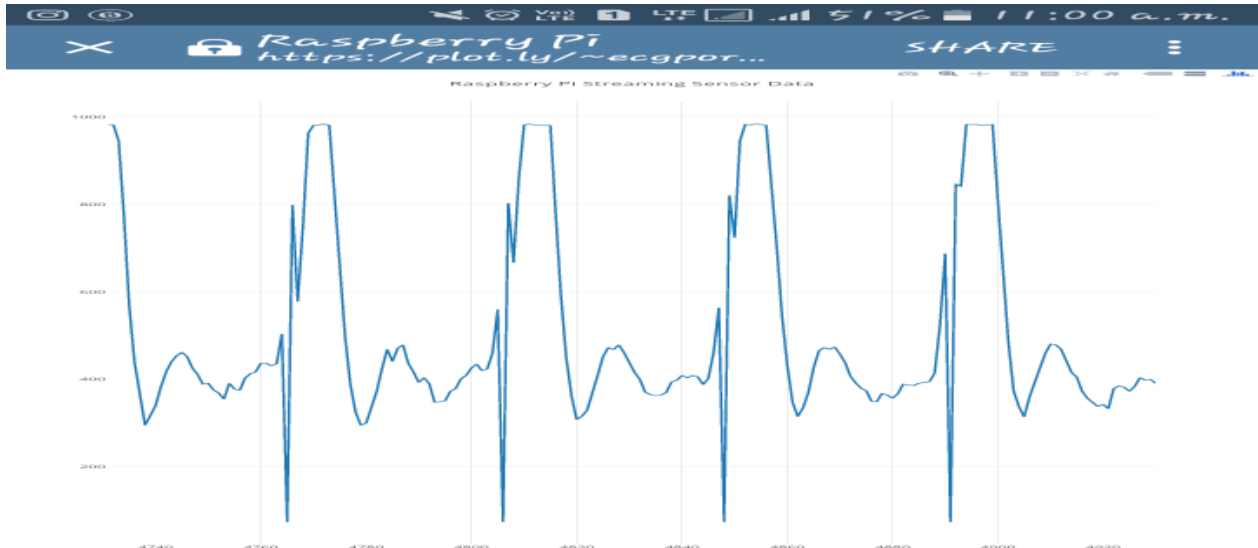

Fig 6.2 ECG output

The above figure is the ECG waveform obtained by the system.

\section{CONCLUSION}

A new wearable device for a healthcare monitoring system was proposed in this paper. The device was implemented in an armband to achieve a non-obstructive system. An armband was chosen as an alternative to previous wearable devices that are strapped to the chest, which can be inconvenient for some users. The technology used in this system uses capacitive coupled electrodes. The capacitive-coupled electrodes enable the system to measure bio-signals through the clothes so that the weaknesses from wet or dry electrodes can be reduced. The bio-signal that was measured in this system is an ECG signal.

Along with the ECG, the bio signals such as temperature, blood pressure, pulse are measured. The system enables us to monitor the real time measurement of these parameters and diagnosis are possible. It has the facilities to record and store the data measured and track the activities of user's heart.Thus using this system, characteristics such as patients' heartbeats, heart conditions, and heart disease can be analyzed.

The proposed wearable arm-band ECG mobile monitoring system has a wide range of applications such as: to check ECG and save the graph in memory, monitor heart attacks, seizures, fainting or collapse, check blood pressure, Measure body temperature, check pulse rate, GPS tracker, inform ambulance and mobile in case of any emergency.

\section{REFERENCES}

[1] H. A. Miller and D. C. Harrison, Biomedical Electrode Technology. New York, NY, USA: Academic, 1974.

[2] mHealth. Available: http://en.wikipedia.org/wiki/MHealth

[3] mHealth Monitoring Diagnostic Medical Devices Market. [Online].Available:http://www.transparencymarketresearch.com/mHealth monitoring-diagnostic-medical-devices.html

[4] mHealth Monitoring and Diagnostic Medical Devices Research. Available: http://www.digitaljournal.com/pr/1877980

[5] Scarle and L. Kirkup, "A direct comparison of wet, dry and insulating bioelectric recording electrodes,” Phys. Meas., vol. 21, no. 2, p. 271,2000.

\section{BIOGRAPHY}

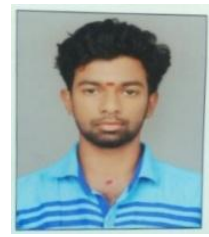

Abijith P M currently pursuing the Bachelor's degree in Applied Electronics \& Instrumentation Engineering from the IES College of Engineering, Thrissur.

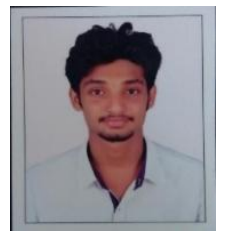

Anshiq Nazar currently pursuing the Bachelor's degree in Applied Electronics \& Instrumentation Engineering from the IES College of Engineering, Thrissur. 
Vol. 5, Issue 4, April 2017

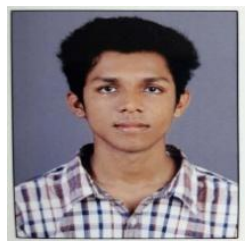

Junain Sidique currently pursuing the Bachelor's degree in Applied Electronics \& Instrumentation Engineering from the IES College of Engineering, Thrissur.

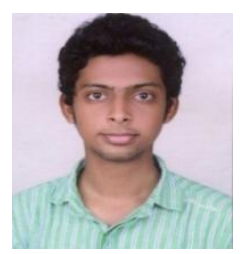

Mohammed Dhanish currently pursuing the Bachelor's degree in Applied Electronics \& Instrumentation Engineering from the IES College of Engineering, Thrissur.

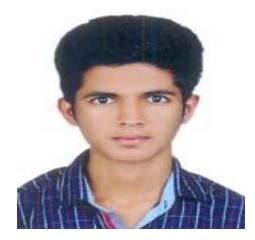

Nuafil Ibrahim currently pursuing the Bachelor's degree in Applied Electronics \& Instrumentation Engineering from the IES College of Engineering, Thrissur.

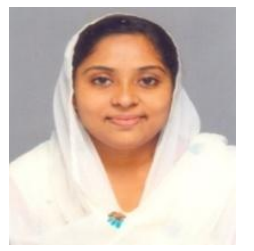

Shahaziya Parvez M completed Master's Degree in Communication Engineering from MG University, Kottayam, and BTech degree from Anna University ,Coimbatore. She is currently working as Assistant Professor in Department of Electronics and Communication Engineering, IES College of Engineering, Thrissur 